\title{
Hard Decisions Shape the Neural Coding of Preferences
}

\author{
Katharina Voigt, ${ }^{1}$ Carsten Murawski, ${ }^{2}$ Sebastian Speer, ${ }^{1,3}$ and $@$ Stefan Bode ${ }^{1,4}$ \\ ${ }^{1}$ Melbourne School of Psychological Sciences, ${ }^{2}$ Department of Finance, The University of Melbourne, Victoria 3010, Australia, ${ }^{3}$ Rotterdam School of \\ Management, Erasmus University, 3000 DR Rotterdam, The Netherlands, and ${ }^{4}$ Department of Psychology, University of Cologne, 50969 Cologne, Germany
}

Hard decisions between equally valued alternatives can result in preference changes, meaning that subsequent valuations for chosen items increase and decrease for rejected items. Previous research suggests that this phenomenon is a consequence of cognitive dissonance reduction after the decision, induced by the mismatch between initial preferences and decision outcomes. In contrast, this functional magnetic resonance imaging and eye-tracking study with male and female human participants found that preferences are already updated online during the process of decision-making. Preference changes were predicted from activity in left dorsolateral prefrontal cortex and precuneus while making hard decisions. Fixation durations during this phase predicted both choice outcomes and subsequent preference changes. These preference adjustments became behaviorally relevant only for choices that were remembered and were in turn associated with hippocampus activity. Our results suggest that preferences evolve dynamically as decisions arise, potentially as a mechanism to prevent stalemate situations in underdetermined decision scenarios.

Key words: choice-induced preference change; decision-making; fMRI; precuneus; preference formation; prefrontal cortex

\section{Significance Statement}

Most theories of decision-making assume that we always choose the best option available, based on a set of stable preferences. However, what happens for hard decisions when the available options are preferred equally? We show that in such stalemate situations, decision-makers adjust their preferences dynamically during the process of decision-making, and these preference adjustments are predicted by a left prefrontal-parietal network. We also show that eye movements during decision-making are predictive of the magnitude of the upcoming value change. Our results suggest that preferences are dynamic, adjusted every time a hard decision is made, prompting a re-evaluation of existing frameworks of decision-making.

\section{Introduction}

Traditional neurocognitive models of value-based choice view decision-making as a serial process in which stable preferences are the basis of subsequent choices (Dolan and Dayan, 2013). However, there are situations in which choice options appear equally valuable to the decision-maker, and therefore existing preferences are not sufficient to rank alternatives. In Jean Buridan's philosophical parable, a hungry donkey is placed between two bales of hay. As alternatives appear equally appealing, the donkey is unable to decide and eventually starves to death. This parable illustrates that there are hard decisions in which existing preferences are not sufficient to identify a preferred option. Instead, preferences might need to be reconstructed dynamically as

Received July 4, 2018; revised Oct. 22, 2018; accepted Nov. 8, 2018.

Author contributions: C.M. and S.B. edited the paper. K.V., C.M., and S.B. designed research; K.V. and S.S. performed research; K.V. analyzed data; K.V. and S.B. wrote the paper.

This study was supported by an Australian Research Council Discovery Early Career Researcher Award (DE 140100350) to S.B. We thank Simon Lilburn and Jacob Paul for helpful discussions, and Sophia Bock, William Turner, and Richard McIntyre for support with MRI data acquisition.

Correspondence should be addressed to Stefan Bode at sbode@unimelb.edu.au.

https://doi.org/10.1523/JNEUROSCI.1681-18.2018

Copyright $\odot 2019$ the authors $\quad 0270-6474 / 19 / 390718-09 \$ 15.00 / 0$ hard decisions arise if we do not want to end up like Burdian's starving donkey.

Indeed, substantial evidence suggests that preferences are not rigid, but evolve dynamically and are dependent on the decision context (Lichtenstein and Slovic, 2006). One highly debated question is whether the act of choosing among equally valued alternatives (henceforth hard decisions) itself shapes preferences. The choice-induced preference change effect refers to the phenomenon that after having made a hard choice, the chosen option is preferred more while the alternative is preferred less (Izuma and Murayama, 2013). Prominent explanations are based on Festinger's (1957) theory of cognitive dissonance, which proposes that discrepancies between actions and preferences cause psychological discomfort. Preferences are then adjusted after a hard decision has been made to reduce the dissonance between initial preference and the decision outcome (for review, see Harmon-Jones et al., 2015). This explanation is in line with neuroimaging studies, which suggested that at the time of reevaluation, after dissonance between preferences and choices is detected by the anterior cingulate cortex (ACC; van Veen et al., 2009; Kitayama et al., 2013), the dorsolateral prefrontal cortex (dlPFC) triggers changes in the neural representation of value 


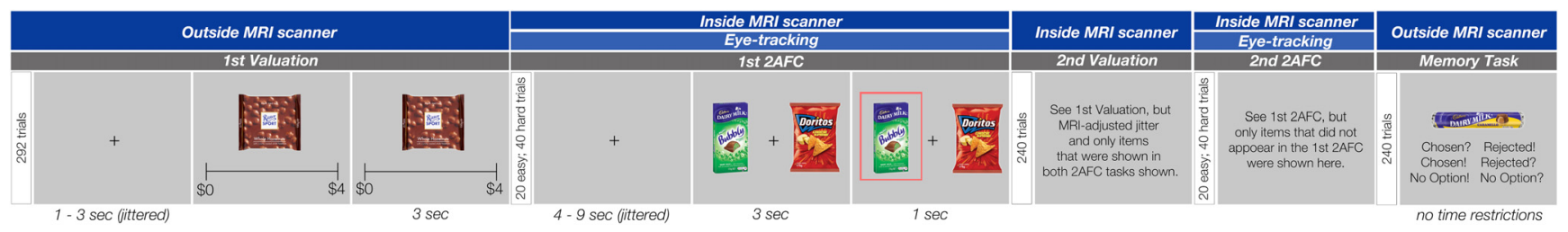

Figure 1. The incentivized free-choice task consisted of four consecutive phases: valuation phase 1, decision phase 1, valuation phase 2, decision phase 2 . This task was followed by a choice memory task.

(Izuma et al., 2010, 2015; Mengarelli et al., 2015) in the ventromedial PFC (vmPFC) or ventral striatum (vStr; Izuma et al., 2010; Chammat et al., 2017).

An alternative possibility is that preferences are adjusted much earlier, that is, while a hard decision is made, when the value differential of the options is not sufficient to choose among them. As such, preference adjustments might constitute a necessary adaptive (online) mechanism to deal with hard choices, as opposed to a post-decisional process for eliminating cognitive dissonance (Izuma et al., 2010, 2015). This new hypothesis, however, remains largely untested as existing functional neuroimaging studies (using methodological improvements of the original paradigm; Chen and Risen, 2010) focused entirely on the neural mechanisms of preference change during re-evaluation (Izuma et al., 2010; Chammat et al., 2017).

Here we tested this hypothesis using the incentive-compatible free choice paradigm (Voigt et al., 2017) while functional magnetic resonance imaging (fMRI) was conducted and eye movements were recorded. We hypothesized that the blood oxygen leveldependent (BOLD) signal in the dlPFC would predict subsequent preference changes during the process of making hard decisions. Other recent studies suggested that post-decisional preference changes only occur when choices are explicitly remembered (Salti et al., 2014), which was associated with hippocampus activity (Chammat et al., 2017). These authors speculate that there could be a causal relationship between episodic memory for previous choices and subsequent changes of preferences. We therefore also predicted similar effects for preference changes during decisionmaking. Additionally, we hypothesized that fixation durations play a significant role in solving hard decisions. Support for this conjecture stems from studies demonstrating that visual fixations causally relate to value-based choices: options that are looked at for longer are more likely to be chosen (Krajbich et al., 2010) and experimental manipulations of exposure duration bias preferences toward the longer presented option (Shimojo et al., 2003). These findings indicate that future values of choice options might be reconstructed by information gathered "in the moment" via fixations.

\section{Materials and Methods \\ Participants}

Thirty male and female human participants were recruited via advertisements at the University of Melbourne. An initial screening interview assured that these participants (1) enjoyed eating snack foods; (2) frequently consumed snack foods; (3) were not on a calorie-, sugar-, or fat-reducing diet; (4) were familiar with local supermarket brands; and (5) had no food allergies. To increase the salience of the experimental stimuli, all participants were asked to refrain from eating or drinking (except for water) for $4 \mathrm{~h}$ before the study (Schonberg et al., 2014; Voigt et al., 2017). Eight participants were excluded from the behavioral and fMRI analyses: five participants arrived too late to finish fMRI data acquisition, for two participants there were technical issues with the track ball used for valuation indication, and one participant revealed only after the experiment that she did not like snack foods and was currently diet- ing. The final sample of 22 participants ( 13 females; aged between 18 and 37 years; $\mathrm{M}=23.57, \mathrm{SD}=4.93$ ) were right-handed (assessed via the Edinburgh handedness inventory; Oldfield, 1971) English speakers with normal or corrected-to-normal vision. An additional screening interview assessing the participants' suitability for MRI revealed that no participant had significant health problems (including neurological and psychiatric disorders) or was on psychoactive medication affecting cognitive function or cerebral blood flow. Participants were naive to the purpose of the study, gave written consent before participating, and were reimbursed AUD60 for their time. The study was approved by the University of Melbourne Human Research Ethics Committee (1442440).

\section{Experimental task and procedures}

The experiment consisted of two main consecutive tasks: the incentivecompatible free choice task (Voigt et al., 2017) followed by a choice memory task. The first task consisted of four task phases: valuation phase 1, decision phase 1 , valuation phase 2 , and decision phase 2 (Fig. 1). Neuroimaging data were acquired during the decision phases and the second valuation phase. Eye-tracking data were acquired during the first decision phase.

\section{The incentivized free-choice task}

Valuation phase 1. Each trial (292 total trials) started with a central fixation cross (jittered between 1 and $3 \mathrm{~s}$ ), which was followed by a pseudorandomly selected snack food stimulus ( $1 \mathrm{~s}$; for further details about these stimuli, see Voigt et al., 2017). Subsequently, participants indicated how much they were willing to pay for that item on a continuum from $\$ 0$ to $\$ 4$. Responses were measured by moving a graphical slider along a continuous valuation scale. All responses were made via a MRI-compatible fiber optic trackball and were restricted to $3 \mathrm{~s}$.

Decision phase 1. A maximum of 80 "hard" and 40 "easy" choice pairs were created, based on the responses of the valuation phase 1, by pairing either items with highly similar (hard) valuations or dissimilar (easy) valuations, respectively (Table 1 ). Half of the hard and easy choice pairs (60 total trials) were shown in a pseudorandomized order, requiring participants to make binary (two-alternative forced-choice; 2AFC) decisions for the item they preferred. Trials were presented in two separate runs (i.e., 30 trials per run) to allow for a short break. Each trial started with a short fixation period (jittered between 4 and $9 \mathrm{~s}$ ), followed by a snack food pair. Critically, it was emphasized that only the item they chose could feature in a subsequent Becker-DeGroot Marshak (BDM auction; Becker et al., 1964). In the BDM auction participants had the chance of buying one of the chosen items, and their willingness to pay (WTP) would serve as the bids from their pre-allocated budget of $\$ 4$. As such, bids and decisions were incentivized and consequential (Voigt et al., 2017). The response window was $3 \mathrm{~s}$ after which the selected choice was highlighted by a black frame for $1 \mathrm{~s}$.

Valuation phase 2. This task phase was identical to the valuation phase 1. Participants were instructed that the purpose was not to probe their memory of the first valuation, but to provide another, independent valuation.

Decision phase 2. This task phase was identical to the first decision phase with the only difference that the remaining, unused 60 choice pairs were presented (Table 1). This allowed us to use the first and second valuations for these items as a control sequence (VVC), assessing changes in valuation that were attributable to methodological artifact pointed out by Chen and Risen (2010). 
Table 1. Descriptive statistics of choice trials in the 2AFC task stages

\begin{tabular}{|c|c|c|c|c|c|c|c|c|c|c|}
\hline & First 2AFC & & & & Second 2 & & & & & \\
\hline & $\begin{array}{l}\text { Average } \\
\text { number }\end{array}$ & $S D_{-a n}$ & $\begin{array}{l}\text { Mean } \\
\text { difference }\end{array}$ & $S D_{-m d}$ & $\begin{array}{l}\text { Average } \\
\text { number }\end{array}$ & $S D_{-a n}$ & $\begin{array}{l}\text { Mean } \\
\text { difference }\end{array}$ & $S D_{-m d}$ & $\begin{array}{l}\text { Paired-samples } \\
t \text { test }\end{array}$ & $p$ \\
\hline Difficult ${ }^{a}$ & 38.69 & 3.92 & 0.003 & 0.01 & 39.07 & 4.13 & 0.003 & 0.02 & $t_{(79)}=-0.77$ & 0.88 \\
\hline Easy $^{b}$ & 19.64 & 1.44 & 2.26 & 1.05 & 19.60 & 0.97 & 2.29 & 1.04 & $t_{(39)}=-0.78$ & 0.88 \\
\hline
\end{tabular}

This table represents the descriptive statistics after choice trial deletion. Average number, Mean number of choice trials across participants; Mean difference, average valuation difference of items within each choice pair across participants; $S D_{-a n}, S D$ for the average number of choice trials; SD_md, SD for mean of valuation difference of items within a choice pair.

${ }^{a}$ Choice trials that contained pairs with a valuation differential of $>1 S D$ of the individual's mean valuation differential were not considered for further analysis.

${ }^{b}$ Choice trials that contained pairs with a valuation differential of $<1 S D$ of the individual's mean valuation differential were not considered for further analysis.

A

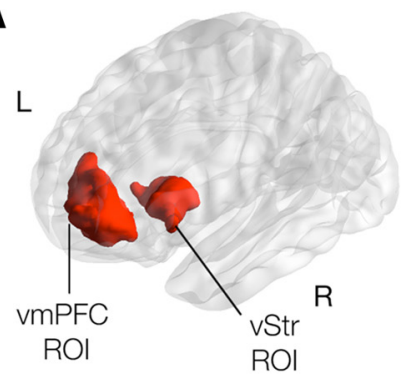

B

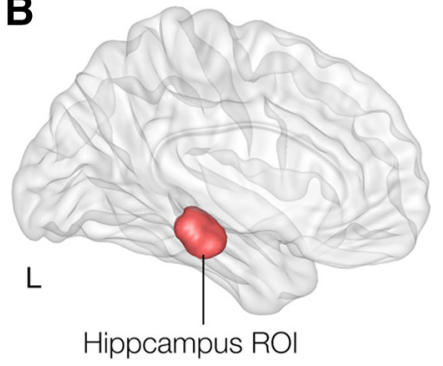

C

$\mathrm{L}$

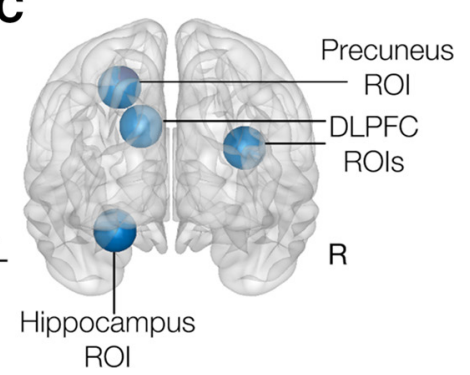

Figure 2. ROls. $\boldsymbol{A}$, The vmPFC and vSTR ROIs were used in GLM1 (based on Bartra et al., 2013, their Fig. 6 A and Table 1). B, The hippocampus ROI was used for GLM 3, GLM 4, and GLM 5. R0I mask in the left hippocampus was created based on Chammat et al. (2017) by using a sphere with $10 \mathrm{~mm}$ radius around their reported peak voxel activation (MNI: $-14,-12,-24)$ in combination with anatomical para- and hippocampal masks from the AAL atlas to ensure that all voxels within the created sphere were within the hippocampal formation. $C$, The precuneus, bilateral dIPFC and hippocampus ROls were used in GLM 5.

The choice memory task

This task was completed outside the MRI scanner. In 292 trials, participants were sequentially presented with all snack foods again. They indicated whether they remembered having previously chosen or rejected it. Critically, participants were asked to distinguish whether they were absolutely certain (options: Chose! or Rejected!; trials labeled "remembered"), or whether they felt that they were guessing (Chose? and Rejected?; trials labeled "guessed") their response. In addition, participants could also indicate that they believed that the item did not feature in the experiment (i.e., No option! or No option?). If participants selected these options, these trials were coded as falsely remembered or falsely guessed. Participants were unaware of the subsequent memory task throughout the fMRI experiment; that is, they were not explicitly instructed to memorize their choices.

\section{Data acquisition and analyses}

\section{Linear mixed modeling of behavioral data}

Choice-induced preference change effects were assessed via linear mixed effects modeling using the Ime4 package (Bates et al., 2015) in R. In the first model, the effects of Condition (coded: $\mathrm{VVC}=0$; VCV $=1$ ), Memory (coded: $0=$ not remembered, remembered $=1$ ), Guessing (coded: $0=$ not guessed, guessed $=1$ ) and the resulting two-way and three-way interactions were regressed on the spread of alternatives, following procedures of previous studies (Chammat et al., 2017). A second linear mixed-effect model aimed to address whether the effect of choice was bidirectional. For this, we added an additional predictor variable to the originally model, i.e., Choice (coded: rejection $=0$; selection $=1$ ) and modeled the (mean-corrected) WTP Change Scores as a function of Choice, Condition, Memory, and Guessing (all variables were coded as mentioned above). In both models, Participants and Stimuli were specified as random intercepts to control for the variance associated with these factors without data aggregation. As choice-induced preference change effects can only be expected for hard decisions (Chen and Risen, 2010; Izuma et al., 2010; Salti et al., 2014; Voigt et al., 2017), the key analyses are reported for hard decision trials only. The final model was established by comparing the full model to alternative models in which model parameters were successively eliminated ("backward elimination"). Model comparison and selection were based on the Bayesian information criterion and log-likelihood ratio testing. The Satterthwaite degrees-of-freedom approximation was used to calculate $p$ values.

\section{fMRI data acquisition preprocessing and analysis}

Whole-brain MRI data were acquired using a Siemens MAGNETOM 3T scanner with a 20-channel head coil (38 axial slices; time of repetition, $\mathrm{TR}=2200 \mathrm{~ms}$; echo time, $\mathrm{TE}=30 \mathrm{~ms}$, resolution $3 \times 3 \times 3 \mathrm{~mm}^{3}$ ). Data acquisition during the two $2 \mathrm{AFC}$ phases comprised of 174 volumes per two runs and 338 volumes per four runs for the valuation phase. Volumes were acquired using a single-shot gradient-echo echoplanar imaging sequence. A high-resolution T1-weighted magnetization-prepared rapid gradient echo covering the whole brain was acquired (TR $=1900 \mathrm{~ms}$; $\mathrm{TE}=2.49 \mathrm{~ms} ; \mathrm{T} 1=900 \mathrm{~ms}$; flip angle $=9^{\circ} ; 192$ slices; field-of-view $=$ $240 \mathrm{~mm}$ ) for each participant. Task-free fMRI data were also acquired before participants performing the task-based fMRI section (results will be reported in a separate publication). The fMRI data were preprocessed using SPM12 (Wellcome Centre for Human Neuroimaging, UCL, UK) software implemented in MATLAB 2014b (MathWorks). Preprocessing included slice time correction, realignment to the individual's mean functional image, coregistration, segmentation, and normalization as well as spatial smoothing (Gaussian kernel's full-width at half-maximum was $8 \mathrm{~mm}$ ).

GLM 1: neural representation of WTP value. To identify brain activity associated with monetary value, we constructed a GLM for the second valuation stage. Results were assessed within regions-of-interests (ROIs) which have been shown to encode stimulus values (Bartra et al., 2013; Fig. 2). This contained two regressors of interests: (1) an onset regressor for the presentation of food stimuli (2) and a parametric regressor for participants' WTP response (ranging from $\$ 0$ to $\$ 4$ ) for that food stimulus:

$$
B O L D_{\text {Valuation2 }}=b_{0}+\text { Stimulus }_{\text {Onset }}+W T P_{\text {Value }}+e .
$$

GLM 2: neural correlates of hard decisions. GLM 2 was designed to establish where in the brain choice difficulty was represented during the crucial first decision phase. The model included two main regressors-ofinterest: (1) onset for hard decisions and (2) onset of easy decisions:

$$
B O L D_{\text {Decision1+2 }}=b_{0}+2 A F C_{\text {OnsetHard }}+2 A F C_{\text {OnsetEasy }}+e .
$$


GLM 3 and 4: memory-depended choice-induced preference changes effects in second valuation phase. Following the logic of the behavioral results, and replicating the exact approach by Chammat et al. (2017), we regressed the interaction effect between experimental condition and correctly remembered choices, which constitutes the hallmark of the preference changes induced by choice, on BOLD signals from the second valuation task stage. To this end, for GLM 3 we sorted trials into four conditions: (1) remembered items in the VCV condition, (2) forgotten items in the VCV condition, (3) remembered items in the VVC condition, and (4) forgotten items in the VCV condition. We regressed the main effect of condition, the main effect of correctly remembered choice outcomes, and the interaction between condition and correctly remembered choice outcomes using a flexible factorial model in the hippocampus ROI (Fig. 2):

$B O L D_{\text {Valuation } 2}=b_{0}+$ Condition + Remembered + Condition

$\times$ Remembered $+e$.

GLM 4 explored the possibility of preference changes for correctly guessed trials. We constructed a similar model as for GLM 3, but with guessed trials only.

$B O L D_{\text {Valuation } 2}=b_{0}+$ Condition + Guessed

$$
+ \text { Condition } \times \text { Guessed }+e .
$$

GLM 5: choice-induced preference change effects during hard choices. Finally, we investigated whether the a priori defined ROI in the dIPFC predicted preference changes on a trial-by-trial basis for hard decisions. For this GLM, we went beyond the model suggested by (Chammat et al. (2017) that only considered the spread of alternatives. To truly capture the choice-induced preference change effect, the WTP valuation change scores (WCS) were entered as a parametric predictor for hard decisions items into the model. WCS were computed by subtracting an item's first WTP score from its second WTP score. Mean-corrected WTP values were used for this subtraction to eliminate any variability in scores that are not attributable to the experimental manipulations. Mean-corrected WTP values were obtained by subtracting the average bid for the respective participant's session from the raw score of each single trial $\left(x_{\mathrm{i}}-\right.$ mean $_{\text {session }}$; Sharot et al., 2009; Salti et al., 2014; Voigt et al., 2017). Easy trials, for which no preference changes were observed and expected, were modeled as additional regressors-of-no-interest:

$B O L D_{\text {Valuation_change }}=b_{0}+$ Decision $_{\text {OnsetHard }}$

$$
+W C S_{\text {Hard }}+\text { Decision }_{\text {OnsetEasy }}+e .
$$

For all GLMs, regressors were convolved with a canonical hemodynamic response and together with the motion parameters from the realignment procedure regressed against the BOLD signal in each voxel. Regressorsof-interest were modeled as independent between levels.

For group-level comparisons, the parameter estimates resulting from the first-level analyses were entered into flexible factorial designs, or repeated measures regressions, depending on the analysis. Appropriate linear contrasts were generated to identify regions associated with the parameter of interest. Results for ROIs were assessed via small volume correction [extent threshold familywise error (FWE), $p<.05$; height threshold $\left.p_{\text {uncorr }}<0.001\right]$. For whole-brain analyses correction for multiple comparisons (extend threshold FWE, $p<0.05$; height threshold, $\left.p_{\text {uncorr }}<0.001\right)$ was performed at cluster level.

Left and right dlPFC ROIs were $10 \mathrm{~mm}$ spheres $(k=155)$ around peak voxel activation associated with preference-changes at the stage of second valuation (i.e., post-choice) reported by Izuma et al. (2010, their Table SI3). Precuneus and hippocampus ROIs were $10 \mathrm{~mm}$ spheres $(k=155)$ around peak voxel activation associated with the Condition $\times$ Remembered (see Fig. $5 D$ ) and Condition $\times$ Guess (see Fig. $5 E$ ) interaction effects observed post-choice in our free-choice paradigm. Note that the hippocampus ROI was again masked with the anatomical para- and hippocampal mask form the automatic anatomical labeling (AAL) atlas. All other ROIs contained voxels of the signified region of $>90 \%$ and therefore additional masking was not necessary.

\section{Eye-tracking acquisition and analysis}

Eye movements were recorded for the right eye for the first choice task stage via an MR-compatible infrared video-based system measuring corneal reflection (Eyelink 1000) at $500 \mathrm{~Hz}$. To assess whether fixation parameters predicted choice, a GLM was conducted. The fixation duration ratio, first and last fixation count of the chosen item were regressed on choice outcome. To determine whether fixation duration predicted updated preferences, we conducted another GLM and regressed the fixation differential (i.e., the mean difference between the fixation duration of chosen and rejected items) and the initial WTP values of the chosen item on the chosen item's WTP values from the second evaluation phase. For this GLM, we analyzed the data from $1000 \mathrm{~ms}$ poststimulus presentation onward, as previous research reported value-formation at this stage (Harris et al., 2011). Analyses were conducted in MATLAB and R.

\section{Results}

\section{Behavioral results}

First, we established the degree of preference changes after individuals made hard choices and their relation to memory of previous choice outcomes. We found that individuals took significantly longer to decide between equally valued alternatives (hard decisions; $\mathrm{M}=1.65 \mathrm{~s}, \mathrm{SD}=0.27 \mathrm{~s}$ ) than between items that were distinct in their values (easy choices; $\mathrm{M}=1.41 \mathrm{~s}, \mathrm{SD}=$ $\left.0.24 \mathrm{~s} ; t_{(21)}=-6.25, p<0.001\right)$. Participants correctly remembered $32.63 \%(\mathrm{SD}=9.41 \%)$ and correctly guessed $25.81 \%(\mathrm{SD}=$ $2.40 \%$ ) of the choice outcomes of their hard choices (difference n.s., $\left.t_{(21)}=1.71, p>0.10\right)$. The interaction between experimental condition (i.e., VCV, VVC) and choice memory is considered diagnostic of a memory-dependent choice-induced preference change effect (Salti et al., 2014; Chammat et al., 2017) such that only correctly remembered items should show preference changes, but not guessed or not remembered items. In a linear mixed effect (LME) model analyses, this interaction showed the hypothesized post-choice spread in valuations for items that were correctly remembered (Condition $\times$ Memory interaction effect: $\beta=0.38, \mathrm{SE}=0.16, p<0.02$ ) but not for correctly guessed items (Condition $\times$ Guessing interaction effect: $\beta=-0.06, \mathrm{SE}=0.09$, $p=0.53$. Replicating our previous behavioral results (Voigt et al., 2017 ), this interaction effect was bidirectional, i.e., WTP increased for correctly remembered chosen items $(\beta=0.26, \mathrm{SE}=$ $0.10, p<0.05)$, and decreased for correctly remembered rejected items $(\beta=-0.17, \mathrm{SE}=0.08, p<0.05$; Fig. 3; Table 2). Replicating previous findings (Sharot et al., 2009; Izuma et al., 2010; Voigt et al., 2017), the choice-induced preference change effect was absent for easy choices (Choice $\times$ Condition $\times$ Remembered interaction effect: $\beta=0.12, \mathrm{SE}=0.17, p>0.05$; Choice $\times$ Condition $\times$ Guess interaction effect: $\beta=0.15, \mathrm{SE}=0.19$, $p>0.05)$.

\section{Neuroimaging results for control analyses \\ Neural representation of monetary value}

We next verified that our WTP procedure engaged the "valuation network", which relates to the encoding of subjective (monetary) value, i.e., preferences (Dolan and Dayan, 2013). To this end, we regressed participants' trial-by-trial WTP scores against BOLD responses during the second valuation phase (GLM1) from ROIs (Fig. 4A) in vmPFC and vStr (Bartra et al., 2013). We found a significant parametric modulation in both the vmPFC (MNI: 3, $\left.50,-4 ; z=4.77 ; p_{\mathrm{SV} . \mathrm{FWE}}<0.001\right)$ and $\mathrm{vStr}$ (MNI: $12,11,-1 ; z=$ $3.59 ; p_{\text {SV.FWE }}=0.036$; Fig. 4 ). 


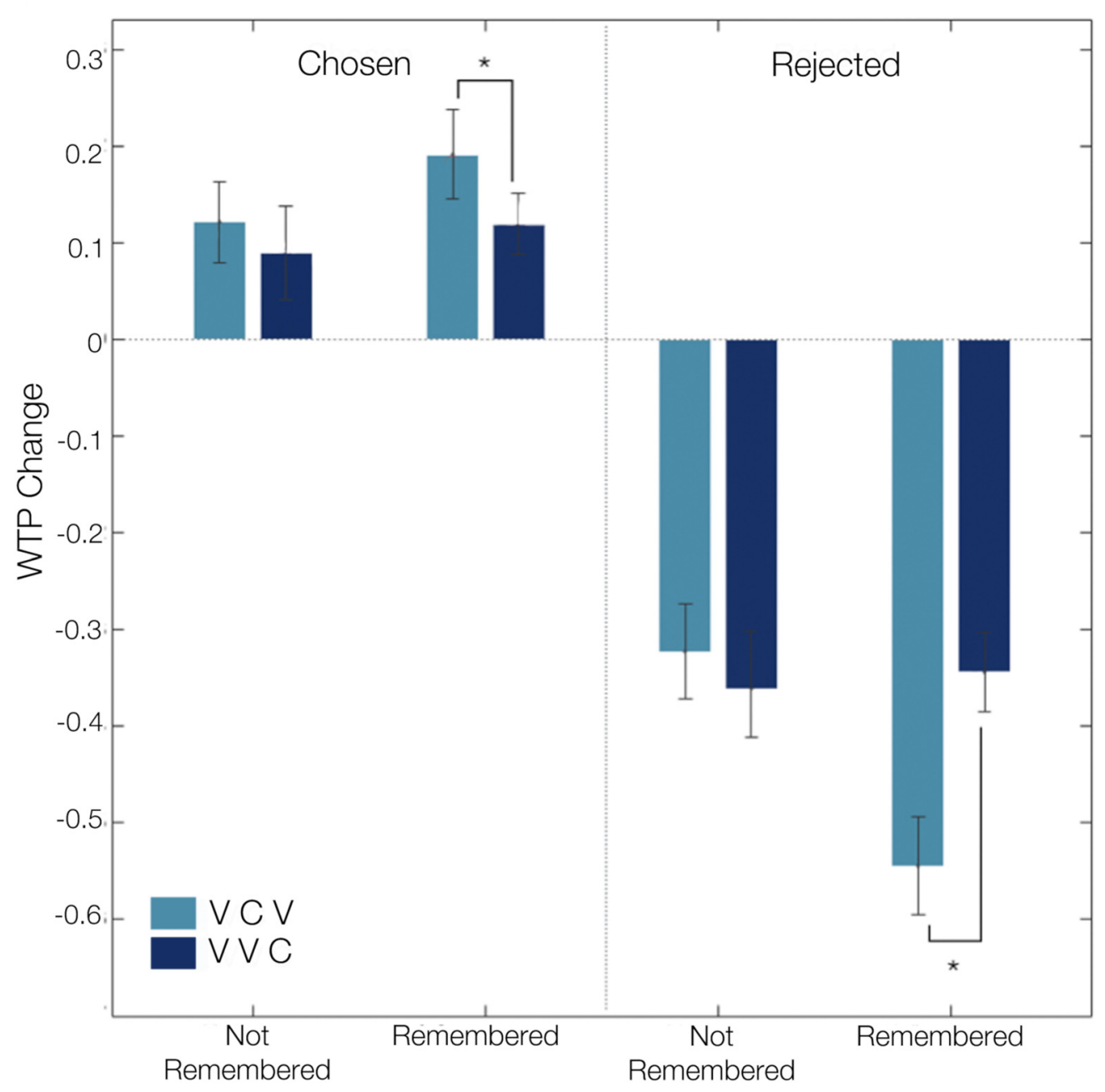

Figure 3. Behavioral results revealed memory-dependent, bidirectional choice-induced preference changes. For chosen/rejected remembered items, the change in WTP values was significantly higher/lower in the VCV condition as opposed to the VVC control condition. This effect did not reach significance for guessed choices. ${ }^{*} p<0.05$.

Table 2. Choice-induced preference change effect for hard decisions

\begin{tabular}{lrr}
\hline & \multicolumn{1}{c}{ Full model } & \multicolumn{1}{c}{ Final model } \\
\hline Intercept) & $-0.26^{* * *}(0.04)$ & $-0.30^{* * *}(0.04)$ \\
Choice & $0.38^{* * *}(0.06)$ & $0.40^{* * *}(0.04)$ \\
Condition & $-0.04(0.05)$ & $-0.01(0.04)$ \\
Remembered & $-0.10(0.06)$ & $-0.06(0.05)$ \\
Guess & $-0.10(0.06)$ & \\
Choice $\times$ Condition & $-0.00(0.08)$ & $-0.00(0.06)$ \\
Choice $\times$ Remembered & $0.10(0.08)$ & $0.07(0.07)$ \\
Condition $\times$ Remembered & $-0.14(0.08)$ & $-0.17^{*}(0.08)$ \\
Choice $\times$ Guess & $0.07(0.09)$ & \\
Choice $\times$ Condition $\times$ Remembered & $0.26^{*}(0.12)$ & $0.26^{*}(0.10)$ \\
Choice $\times$ Condition $\times$ Guess & $-0.00(0.12)$ & \\
AIC & 7557.29 & 7538.23 \\
BIC & 7649.48 & 7605.84 \\
Log likelihood & -3763.65 & -3758.11 \\
\hline
\end{tabular}

AIC, Akaike information criterion; BIC, Bayesian information criterion. The baseline condition is Rejected, VVC, Remembered Wrong, Guess Wrong. Conditions are treatment coded. ${ }^{* *} p<0.001,{ }^{*} p<0.05$.

Neural correlates of hard choices

We then confirmed that hard choices activated decision-related brain regions to a greater extent than easy choices (GLM2), suggesting decision conflict. A whole-brain analysis showed that activity in the left dorsal ACC [MNI: $-6,26$, 4; extent threshold $p_{\text {SV.FWE }}=0.006\left(p_{\text {uncorr. }}<0.001\right.$, height threshold $) ; z=4.21 ; k=$
130] and left middle frontal gyrus [MFG; MNI: $-45,23,26$; extent threshold $p_{\text {SV.FWE }}=0.04$ ( $p_{\text {uncorr. }}<0.001$ height threshold); $z=3.91 ; k=79$ ] was significantly higher for hard compared with easy choices (Fig. 4B). These regions have previously been related to decisions between equally valued options, approach conflicts, and choice anxiety (van Veen et al., 2009; Kitayama et al., 2013; Shenhav and Buckner, 2014).

\section{Preference changes following hard decisions}

Neuroimaging results

Next, we investigated the neural correlates of the memorydepended choice-induced preference changes at the time of reevaluation. As a recent study (Chammat et al., 2017) provided initial evidence that this effect is associated with left hippocampus activity, we used their results to construct a ROI. Consistent with our behavioral results, the predicted critical interaction between experimental condition and remembered choice outcomes was associated with changes in left hippocampus activity (MNI: -24 , $-28,-16 ; p_{\text {SV.FWE }}=0.012 ; F=12.56, z=3.18$; Fig. $\left.5 D\right)$. An additional whole-brain analysis confirmed that no other brain regions showed this effect. Although the interaction between correctly guessed choice outcomes and experimental condition did not show any change in preference at the behavioral level, significant effects for correctly guessed items were found at the wholebrain level in one cluster within left posterior parietal cortex, 
A Neuronal Representation of Monetary Value
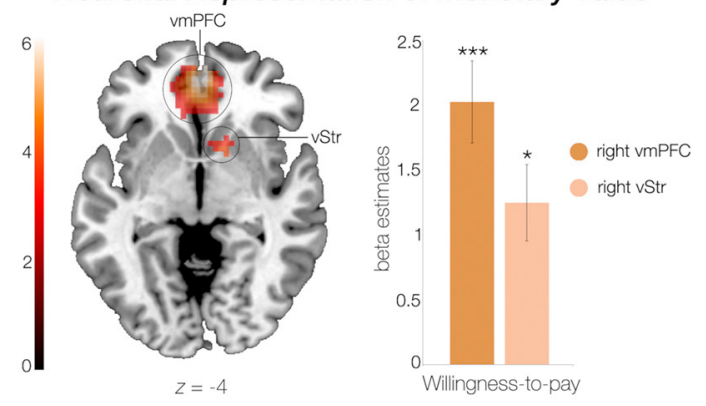

B Neuronal Correlates of Difficult Value-Based Choice
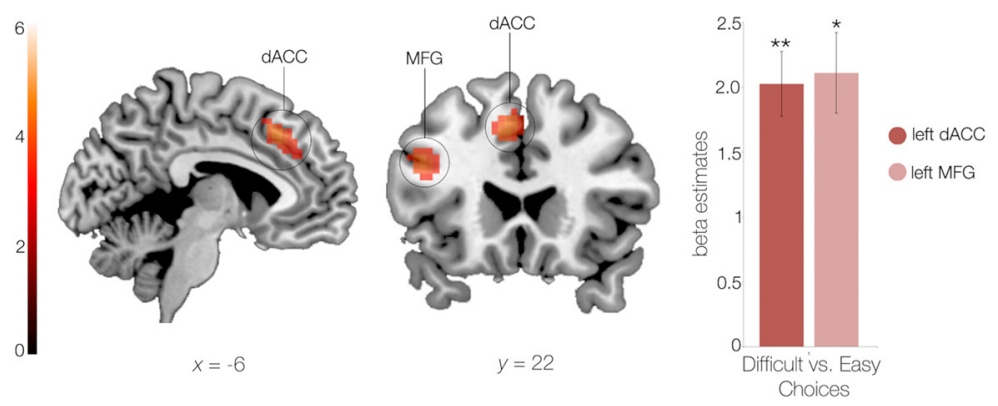

Figure 4. Brain areas encoding monetary value and value-based choices between equal-value options. $A$, Monetary values (i.e., WTP scores) were parametrically associated with the BOLD signal in a priori defined regions of interest in the VmPFC and VStr. $B$, The dACC and the MFG showed greater BOLD signals during hard decisions compared with easy decisions. Color bar indicates $z$-statistic of parameter estimates. Bar graphs represent averaged parameter estimates. Error bars indicate SEM. ${ }^{*} p<0.05 ;{ }^{* *} p<0.01 ;{ }^{* * *} p<0.001$.

Preference changes during hard decisions

Eye-tracking Results

A Fixation duration predicts choices

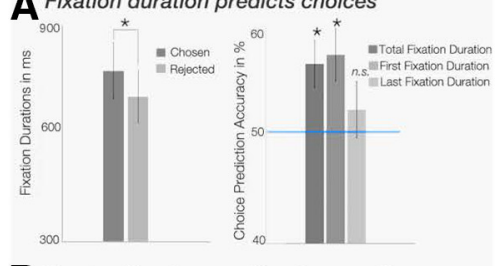

BFixation duration predicts future values

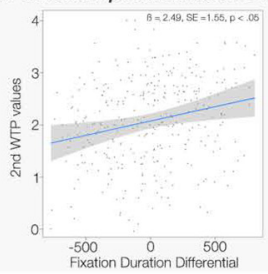

fMRI Results

CBrain areas associated with trial-by trial value change

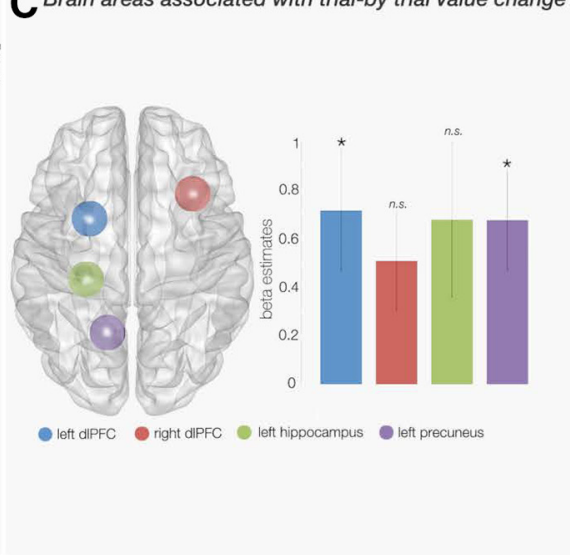

Preference changes after hard decisions

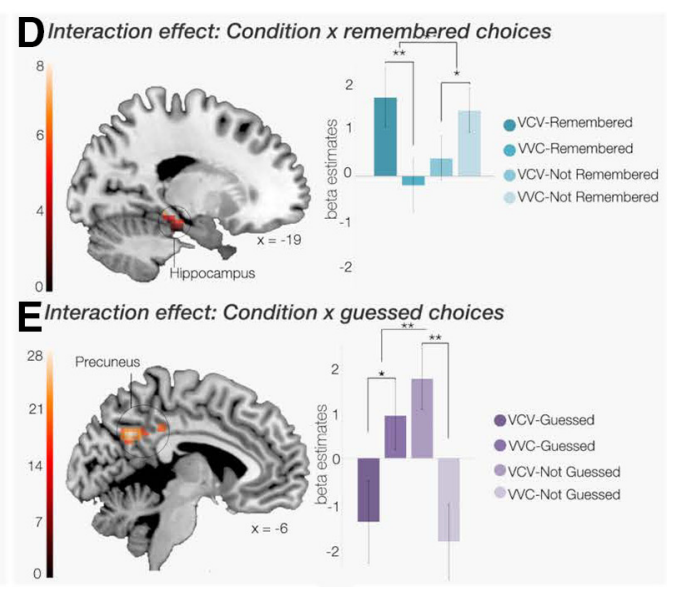

Figure 5. Preference formation during and following hard choices. In-choice results: $A$, Fixation Duration for chosen and rejected items. $B$, Choice prediction accuracy of total, first and last fixation duration. $\boldsymbol{B}$, Fixation duration differential (i.e., fixation duration of chosen items minus fixation duration of rejected item) predicted upcoming valuation change of chosen item. $C$, Brain regions of interest associated with trial-by-trial preference change at the time of decision-making between equally valued items. Post-choice results: $\boldsymbol{D}$, The interaction effect between experimental condition and correctly remembered choice outcomes was associated with left hippocampus activity. $E$, The interaction effect between experimental condition and correctly guessed choice outcomes was associated with left hippocampus activity. ${ }^{*} p<0.05 ;{ }^{* *} p<0.01$.

including the precuneus [MNI: $-6,-55,32$; extent threshold $p_{\text {SV.FWE }}=0.01\left(p_{\text {uncorr. }}<0.001\right.$ height threshold $) ; z=4.61 ; k=$ 133; Fig. 5E], but not in the hippocampus.

\section{Preference changes during hard decisions}

Neuroimaging results

Our crucial analyses involved testing whether preference changes were already present at a neural level during the process of making hard decisions, and whether these effects were moderated by memory processes, as suggested by our behavioral results. Previous studies suggested that the dlPFC (Izuma et al., 2010, 2015), particularly the left (Harmon-Jones et al., 2015; Mengarelli et al., 2015), is directly involved in post-decisional preference changes. We therefore regressed trial-by-trial preference change scores for each of the hard choices as a parametric regressor against the BOLD data obtained during the first decision phase (GLM 5) from predefined ROIs in the left and right dIFPC (Fig. $5 C$; Izuma et al., 2010). Our analysis showed that activity in the left dlPFC was predictive of subsequent preference changes for the later remembered items (MNI: $-24,5,47 ; p_{\mathrm{SV} . \mathrm{FWE}}=0.01, z=3.61$ ). The same contrast in right dIPFC ROI marginally missed the significance threshold (MNI: 33, 20, 30; $p_{\mathrm{SV} . \mathrm{FWE}}=0.07, z=$ 2.80).

As we found choice-induced preference changes to be encoded in hippocampus (remembered) and precuneus (guessed) for the second valuation phase, we further repeated the above analysis (GLM 5) for the decision phase using these areas as ROIs. This analysis showed that the precuneus (MNI: $-15,-58,29$; $\left.z=3.59 ; p_{\mathrm{SV} . \mathrm{FWE}}=0.008\right)$, but not hippocampus (MNI: -27 , $\left.-28,-19 ; z=2.03 ; p_{\mathrm{SV} . \mathrm{FWE}}=0.48\right)$ encoded the preference changes at the time of decision-making (Fig. $5 C$ ).

To assess whether the effects were specific to the choice task stage in which choice can shape preferences (i.e., VCV), we repeated the analyses using the data obtained during second choice task stage (i.e., VVC). We did not find any significant results for any of the ROIs, indicating that the results were unique neural correlates of preference changes induced by choice and not artifacts because of regression to the mean.

Eye tracking results

Our neuroimaging results do not address the question whether preferences changed as a consequence of choice, or alternatively, 
whether they changed during the decision-making process. To answer this question, we analyzed fixation data during the decision-making process (in the same phase 1) while participants were exposed to both options on the screen preceding their response.

Decision outcomes. The total fixation duration for chosen items $(\mathrm{M}=693.59 \mathrm{~ms}, \mathrm{SD}=253.34 \mathrm{~ms})$ was significantly higher than for rejected items $\left(\mathrm{M}=634.65 \mathrm{~ms}, \mathrm{SD}=232.80 \mathrm{~ms} ; t_{(14)}=\right.$ $3.48, p=0.004 ; d=0.89)$. Similarly, the fixation duration ratio for chosen items $(\mathrm{M}=0.44, \mathrm{SD}=0.15)$ was significantly higher than the fixation duration ratio for rejected items $(M=0.40$, $\mathrm{SD}=0.14 ; t_{(14)}=2.89, p=0.003 ; d=1.06$; Fig. $5 A$ ). The fixation duration ratio was computed by dividing the fixation duration for the chosen item (or rejected item, respectively) by the total fixation duration (for both the chosen and rejected item). A linear regression model showed that the probability of choosing an item was determined by its fixation duration ratio $(\beta=0.07$, $\mathrm{SE}=0.03, p<0.05)$, but also by whether it was looked at first $(\beta=0.28, \mathrm{SE}=0.14, p<0.05)$ and last $(\beta=0.37, \mathrm{SE}=0.14, p<$ $0.01)$. Both the accuracies with which choices could be predicted from the total fixation duration $(\mathrm{M}=0.56, \mathrm{SD}=0.08)$ and the duration of the first fixation $(\mathrm{M}=0.57, \mathrm{SD}=0.09)$ were significantly higher than chance (total fixation duration: $t_{(14)}=2.91$, $p=0.01 ; d=0.75$; first fixation duration: $t_{(14)}=2.94, p=0.01$; $d=0.76)$. Fixation duration of the last fixation, however, did not predict choice outcomes $\left(\mathrm{M}=0.52, \mathrm{SD}=0.1 ; t_{(14)}=0.72, p=\right.$ 0.48; Fig. 5A).

Preference changes. Next, we examined whether fixation durations also predicted subsequent changes in valuation for hard choices during the first decision phase. A LME model showed that the fixation duration differential (i.e., the mean difference between the fixation duration of chosen and rejected items) predicted the subsequent, updated WTP value for the chosen item ( $\beta=5.55, \mathrm{SE}=1.96, p<0.001$, one-tailed $)$, with higher fixation rates being linked to higher updated values, as early as $1000 \mathrm{~ms}$ after stimulus presentation. This finding also held when controlling for the initial WTP values $(\beta=2.49, \mathrm{SE}=1.55, p<0.05$, one-tailed; Fig. $5 B$ ). The analysis revealed no interaction effect with item memory.

\section{Discussion}

Preference changes following hard choices have been interpreted as a result of cognitive dissonance reduction after decisions have been made and preferences are re-assessed (Festinger, 1957). As such, previous fMRI studies of preference changes induced by hard choices solely focused on neural correlates of preference changes during the re-evaluation of alternatives (Izuma et al., 2010; Chammat et al., 2017). Our study is the first to reveal that preference changes were linked with neural activity much earlier, i.e., during the process of making hard decisions. Specifically, activation in a brain network comprising left dlPFC and precuneus was predictive of upcoming preference changes effects. Further, we found that fixation durations predicted choices as well as future valuations. Although our findings do not rule out the contribution of cognitive dissonance reduction after decisionmaking, our results clearly point to the involvement of a profoundly different mechanism of preference change in which preferences are adjusted online in the early stages of the decision process while decision-makers are deciding among equally valued alternatives.

Our behavioral results confirmed that for equally valued, consumable items, the valuations increased after choosing and decreased after rejecting when valuations were measured by incentive-compatible WTP assessments (Voigt et al., 2017). Consistent with other earlier reports (Salti et al., 2014; Chammat et al., 2017) we confirmed that preferences changed only for choices that were explicitly remembered later. Our memory test explicitly addressed the possibility that participants did not actually remember which choices they made earlier, but simply inferred (i.e., guessed) them based on their updated preferences. By asking participants to label their choice outcomes as remembered or guessed we could show that explicit choice memory, but not correct guessing, was linked to behavioral choice-induced preference change effects, fully in line with Chammat et al. (2017). We further replicated findings by Chammat et al. (2017) that memory-depended choice-induced preference changes were associated with left hippocampus activity, a core region involved in long-term episodic memory (Bird and Burgess, 2008), during the revaluation phase. In our study, the same neural correlates of the spread of alternatives was only found for remembered items. In addition, while preference changes were absent at the behavioral level for correct guesses, a significant neural effect for spread of alternatives in this condition could nevertheless be observed in the precuneus. This region has been associated with the rapid formation and retrieval of episodic memory (Brodt et al., 2016), with self-relevant processing (Kircher et al., 2002) and with decisions based on guessing (Bode et al., 2013). The precuneus might therefore be involved in less certain retrieval processes for items, which only lead to smaller, behaviorally subthreshold choiceinduced preference change effects.

Previous explanations for the observed spread of alternatives following hard choices (Izuma et al., 2010; Salti et al., 2014; Chammat et al., 2017) were in accord with prominent theories that valuations are adjusted post hoc to match previous choices (e.g., cognitive dissonance theory, Festinger, 1957; self-perception theory, Bem, 1967). However, the temporal dynamics of choiceinduced preference changes were never explicitly tested. Some other studies attempted to investigate whether preference change during the initial decision phase (Jarcho et al., 2011; Kitayama et al., 2013; Colosio et al., 2017) but remained inconclusive as they did not distinguish decision conflict from preference change (Colosio et al., 2017), did not control for potential methodological artifacts (cf., Chen and Risen, 2010), or used noisy, incentiveincompatible preference assessments, which might be ill-suited to investigate choice-induced preference changes (cf., Voigt et al., 2017). Our study accounted for these methodological issues and clearly showed that trial-by-trial preference changes were already reflected in the dlPFC during the decision process. The left dlPFC has been shown previously to be involved in the implementation of preference change after hard choices were made (Izuma et al., 2010; Mengarelli et al., 2015). Here, we extended these findings in showing that this area was involved much earlier. In addition, we tested whether the memory-related regions, which were found to reflect the spread of alternatives (as conceptualized by Chammat et al., 2017) in our study in the revaluation phase also tracked changes in preferences during decision-making. Interestingly, such effects were absent in the hippocampi but present in the precuneus.

Together, our fMRI results suggest a process in which first, a decision conflict among equally valued alternatives is detected, based on subjective values of the choice alternatives. In line with this, monetary values were associated with activity in vmPFC and vStr in our study (cf., Bartra et al., 2013), whereas decision conflict was reflected in enhanced activity in the ACC and MFG (cf., Botvinick, 2007; Shenhav and Buckner, 2014). The detection of decision conflict could then trigger an updating process for stim- 
ulus values during decision formation, possibly to resolve the initial conflict and to avoid similar near-stalemate situations in the future. This idea is consistent with a revised version of cognitive dissonance theory (Harmon-Jones et al., 2015), which states that a decision conflict needs to be resolved first to enable the individual to prepare a choice plan. This process could therefore involve the dlPFC, which is strongly related to decision-making and working memory (Yan et al., 2016) as well as the precuneus, which might be more involved in the initial formation of episodic memory and potentially driving self-referential decision processes via allocation of attention (Kircher et al., 2002; Brodt et al., 2016). Shifts in spatial attention related to precuneus activity during decision formation could then feed into the reconstruction of new value information in the dlPFC, which in turn could store the new value representation in working memory, assisting the optimal decision between the options. This is in line with demonstrations that value reconstruction evolves from posterior parietal to dorsolateral prefrontal regions (Harris et al., 2011). Consequently, during subsequent revaluation, stronger changes in preference, and also stronger memory-related signals, would be found for the same items, which is what we and others (Salti et al., 2014; Chammat et al., 2017) observed. These authors suggested that the function of memory for choice-induced preference change effects might be based on a metacognitive mechanism regulating the coherence between our previous remembered decisions and current beliefs, values and behaviors at revaluation. Our finding of preference change prediction during choice appears partly at odds with this account and rather suggests an immediate, automatic and unconscious updating process. Differences in familiarity (Dubé et al., 2013), or fluctuations in attention (Chun and Turk-Browne, 2007) between items could be related to both preference update during choice as well as encoding strength. Hence, it is possible that the same metacognitive mechanism proposed by Chammat et al. (2017) already unconsciously operates during choice, consolidating stronger memory traces for updated items that are then consciously accessible during revaluation. Other items, labeled as "forgotten" later, might only receive small updates which are negligible or remain undetected. In consequence, preference updating processes during and post-choice might coexist, but their interplay with memory functions requires further research.

It has to be noted, however, that ultimately the role of dlPFC (and of the other brain regions reported here) in preparing preference changes during choice cannot be fully resolved. It remains possible that other processes, strongly correlated with computing updated preferences, drove the activation profile in dlPFC during the decision phase. Given the current literature, however, the suggested model constitutes a reasonable assumption.

To investigate whether preferences were indeed reconstructed during decision formation for hard decisions, we also analyzed fixations via eye tracking. Previous studies postulated a causal link between visual fixations and the formation of subjective values and value-based choice (Shimojo et al., 2003; Krajbich et al., 2010). According to these studies, the allocation of spatial attention might lead to an increase in information accumulation in favor for the fixated object and, in turn, a higher likelihood of this object to be chosen. In accordance with these reports, we found that fixation duration of the first fixation and total fixation duration predicted choices. As such, a proportion of these signals could potentially reflect residual preference differences between choice options. However, beyond choice itself, fixations also predicted changes in future values for chosen items. This analysis could not explicitly take into account methodological artifacts pointed out by Chen and Risen (2010), but given that we demonstrated clear choice-induced preference change effects after controlling for such effects for the same items, this strongly suggests that our findings did indeed reflect preference adjustments during hard decision, supporting the neural findings for the decision phase. These adjustment processes potentially also drive the encoding of choice options in episodic memory, meaning that subsequent choice memory might not reflect random variations in retrieval strength, but systematic differences in encoding strength during decision formation.

\section{The formation of decisions and preferences}

Our results cannot unambiguously disentangle which processes contribute to making the decision versus adjusting preferences. As early fixations predicted choices, it is possible that these fixations reflect allocation of attention, potentially acting as a "symmetry breaker" when confronted with equally valuable options. This could be driven by the precuneus, which has been related to choices under indifference (Bode et al., 2013; Soon et al., 2013). The precuneus also has major subcortical connections to the pretectal area and the superior colliculus (Yeterian and Pandya, 1993; Leichnetz, 2001), which contribute to attentional shifts via eye movement control (Moschovakis, 1996). However, early fixations patterns must not necessarily be the result of random processes, but could themselves be driven by exogenous stimulus properties, such as its saliency (Itti and Koch, 2001), or residual differences in subjective value, which were not adequately captured by our WTP measurements. Further, it is possible that for the longer fixated item, more choice-attributes were considered, leading to a choice-advantage and preference increase (Orquin and Mueller Loose, 2013). Alternatively, the mere exposure effect (Zajonc, 1968), which states that preferences increase as a function of exposure duration, might also be relevant here.

In conclusion, our findings indicate that theories that focus on post-decisional factors cannot be the sole explanation of choiceinduced preference effects. Our results support a dynamic view of preference formation during decision-making, enabling the individual to make value-based choices. The overall preference change could nevertheless result from a combination of adjustment processes during and following choice, and the extent to which each mechanism contributes to the effect remains an open question. Future studies are also needed to explore what factors underlie the early fixations, which predicted both choice and updated preferences, and whether similar effects can be found in choices other than value-based decisions.

\section{References}

Bartra O, McGuire JT, Kable JW (2013) The valuation system: a coordinatebased meta-analysis of bold fMRI experiments examining neural correlates of subjective value. Neuroimage 76:412-427. CrossRef Medline

Bates D, Mächler M, Bolker B, Walker S (2015) Fitting Linear Mixed-Effects Models Using lme4. J Stat Softw 67:1-48. CrossRef

Becker GM, DeGroot MH, Marschak J (1964) Measuring utility by a singleresponse sequential method. Behav Sci 9:226-232. CrossRef Medline

Bem DJ (1967) Self-perception: An alternative interpretation of cognitive dissonace phenomena. Psychol Rev 74:183-200. CrossRef

Bird CM, Burgess N (2008) The hippocampus and memory: insights from spatial processing. Nat Rev Neurosci 9:182-194. CrossRef Medline

Bode S, Bogler C, Haynes JD (2013) Similar neural mechanisms for perceptual guesses and free decisions. Neuroimage 65:456-465. CrossRef Medline

Botvinick MM (2007) Conflict monitoring and decision making: reconciling two perspectives on anterior cingulate function. Cogn Affect Behav Neurosci 7:356-366. CrossRef Medline

Brodt S, Pöhlchen D, Flanagin VL, Glasauer S, Gais S, Schönauer M (2016) Rapid and independent memory formation in the parietal cortex. Proc Natl Acad Sci U S A 113:13251-13256. CrossRef Medline 
Chammat M, Karoui IE, Allali S, Hagège J, Lehongre K, Hasboun D, Baulac M, Epelbaum S, Michon A, Dubois B, Navarro V, Salti M, Naccache L (2017) Cognitive dissonance resolution depends on episodic memory. Sci Rep 7:41320. CrossRef Medline

Chen MK, Risen JL (2010) How choice affects and reflects preferences: revisiting the free-choice paradigm. J Pers Soc Psychol 99:573-594. CrossRef Medline

Chun MM, Turk-Browne NB (2007) Interactions between attention and memory. Curr Opin Neurobiol 17:177-184. CrossRef Medline

Colosio M, Shestakova A, Nikulin VV, Blagovechtchenski E, Klucharev V (2017) Neural mechanisms of cognitive dissonance (revised): An EEG study. J Neurosci 37:5074-5083. CrossRef Medline

Dolan RJ, Dayan P (2013) Goals and habits in the brain. Neuron 80:312325. CrossRef Medline

Dubé C, Payne L, Sekuler R, Rotello CM (2013) Paying attention to attention and recognition memory: insights from models and electrophysiology. Psychol Sci 24:2398-23408. CrossRef Medline

Festinger L (1957) A theory of cognitive dissonance. New York: Row Peterson.

Harmon-Jones E, Harmon-Jones C, Levy N (2015) An action-based model of cognitive-dissonance processes. Curr Dir Psychol Sci 24:184-189. CrossRef

Harris A, Adolphs R, Camerer C, Rangel A (2011) Dynamic construction of stimulus values in the ventromedial prefrontal cortex. PLoS One 6:e21074. CrossRef Medline

Itti L, Koch C (2001) Computational modelling of visual attention. Nat Rev Neurosci 2:194-203. CrossRef Medline

Izuma K, Matsumoto M, Murayama K, Samejima K, Sadato N, Matsumoto K (2010) Neural correlates of cognitive dissonance and choice-induced preference change. Proc Natl Acad Sci U S A 107:22014-22019. CrossRef Medline

Izuma K, Murayama K (2013) Choice-induced preference change in the freechoice paradigm: a critical methodological review. Front Psychol 4:41. CrossRef Medline

Izuma K, Akula S, Murayama K, Wu DA, Iacoboni M, Adolphs R (2015) A causal role for posterior medial frontal cortex in choice-induced preference change. J Neurosci 35:3598-3606. CrossRef Medline

Jarcho JM, Berkman ET, Lieberman MD (2011) The neural basis of rationalization: cognitive dissonance reduction during decision-making. Soc Cogn Affect Neurosci 6:460-467. CrossRef Medline

Kircher TT, Brammer M, Bullmore E, Simmons A, Bartels M, David AS (2002) The neural correlates of intentional and incidental selfprocessing. Neuropsychologia 40:683-692. CrossRef Medline

Kitayama S, Chua HF, Tompson S, Han S (2013) Neural mechanisms of dissonance: an fMRI investigation of choice justification. Neuroimage 69:206-212. CrossRef Medline

Krajbich I, Armel C, Rangel A (2010) Visual fixations and the computation and comparison of value in simple choice. Nat Neurosci 13:1292-1298. CrossRef Medline

Leichnetz GR (2001) Connections of the medial posterior parietal cortex (area 7m) in the monkey. Anat Rec 263:215-236. CrossRef Medline

Lichtenstein S, Slovic P (2006) The construction of preference. New York: Cambridge UP.

Mengarelli F, Spoglianti S, Avenanti A, di Pellegrino G (2015) Cathodal tDCS over the left prefrontal cortex diminishes choice-induced preference change. Cereb Cortex 25:1219-1227. CrossRef Medline

Moschovakis AK (1996) The superior colliculus and eye movement control. Curr Opin Neurobiol 6:811-816. CrossRef Medline

Oldfield RC (1971) The assessment and analysis of handedness: the Edinburgh inventory. Neuropsychologia 9:97-113. CrossRef Medline

Orquin JL, Mueller Loose S (2013) Attention and choice: a review on eye movements in decision making. Acta Psychol 144:190-206. CrossRef Medline

Salti M, El Karoui I, Maillet M, Naccache L (2014) Cognitive dissonance resolution is related to episodic memory. PLoS One 9:e108579. CrossRef Medline

Schonberg T, Bakkour A, Hover AM, Mumford JA, Nagar L, Perez J, Poldrack RA (2014) Changing value through cued approach: an automatic mechanism of behavior change. Nat Neurosci 17:625-630. CrossRef Medline

Sharot T, DeMartino B, Dolan RJ (2009) How choice reveals and shapes expected hedonic outcome. J Neurosci 29:3760-3765. CrossRef Medline

Shenhav A, Buckner RL (2014) Neural correlates of dulling affective reactions to win-win choice. Proc Natl Acad Sci U S A 111:10978-10983. CrossRef Medline

Shimojo S, Simion C, Shimojo E, Scheier C (2003) Gaze bias both reflects and influences preferences. Nat Neurosci 6:1317-1322. CrossRef Medline

Soon CS, He AH, Bode S, Haynes JD (2013) Predicting free choices for abstract intentions. Proc Natl Acad Sci U S A 110:6217-6222. CrossRef Medline

van Veen V, Krug MK, Schooler JW, Carter CS (2009) Neural activity predicts attitude change in cognitive dissonance. Nat Neurosci 12:14691474. CrossRef Medline

Voigt K, Murawski C, Bode S (2017) Endogenous formation of preferences: choices systematically change willingness-to-pay for goods. J Exp Psychol Learn Mem Cogn 43:1872-1882. CrossRef Medline

Yan Y, Wei R, Zhang Q, Jin Z, Li L (2016) Differential roles of the dorsal prefrontal and posterior parietal cortices in visual search: a TMS study. Sci Rep 6:30300. CrossRef Medline

Yeterian EH, Pandya DN (1993) Striatal connections of the parietal association cortices in the rhesus monkey. J Comp Neurol 332:175-197. CrossRef Medline

Zajonc RB (1968) Attitudinal effects of mere exposure. J Pers Soc Psychol 9:1-27. CrossRef 